\title{
The unfavorable influence of the prematurity on the neonatal prognostic of small for gestational age fetuses
}

\author{
Roxana Bohiltea ${ }^{1}$, Natalia Turcan ${ }^{2}$, Florentina Furtunescu ${ }^{3}$, Dan Navolan ${ }^{4}$, and Monica \\ Cirstoiu $^{3}$ \\ ${ }^{1}$ Universitatea de Medicina si Farmacie Carol Davila Facultatea de Medicina \\ ${ }^{2}$ University Emergency Hospital Bucharest \\ ${ }^{3}$ Carol Davila University of Medicine and Pharmacy Faculty of General Medicine \\ ${ }^{4}$ Universitatea de Medicina si Farmacie Victor Babes din Timisoara
}

May 5, 2020

\begin{abstract}
Introduction. Vascular stress at the level of the uterus-placental unit, with chronic placental ischemia, results in intrauterine growth restriction. Expectation management can be used, when the situation allows, in cases of compensated intrauterine growth restriction. Aim: The aim of our study was to evaluate the neonatal prognosis of preterm births with and without growth restriction and term births with growth restriction in order to improve decisional accuracy regarding the termination of pregnancy. Results: The frequency of term birth infants with low birth weight for gestational age was approximately $2 \%$. The male gender, predominated only in the group of premature infants with normal weight for the gestational age. The highest frequency of neonatal complications studied occurred in the group of preterm neonates SGA (small for gestational age) with statistical significance obtained for cardio-vascular arrest acute respiratory failure, ulcer-necrotic enterocolitis, respiratory distress, cerebral edema, intraventricular hemorrhage, cerebral hemorrhage, pulmonary hemorrhage, neonatal infection, hypoglycemia, retinopathy, anemia, hemorrhagic disease, disseminated intravascular coagulation, disease of hyaline membranes, neonatal sepsis, need for intensive neonatal therapy and death. Conclusion: Immediate neonatal adaptation of SGA preterm neonates is more deficient than for preterm neonates with appropriate weight for gestational age; the adaptation of preterm neonates, in turn, is more deficient than term newborns with intrauterine growth restriction. The term newborns with intrauterine growth restriction have a neonatal adaptation comparable to that of the term newborns with weight corresponding to the gestational age.
\end{abstract}

Key words: Intrauterine growth restriction, cerebroplacental ratio, prematurity

\section{Introduction}

Fetal consequences of defective placentation with poor vascular flow secondary to abnormal physiological transformation of the spiral arteries are intrauterine growth restriction, oligohydramnios, abruptio placentae, and adverse fetal biophysical score. In this context, the fetuses resulting from pre-eclampsia pregnancies have an increased risk of preterm birth and unfavorable perinatal and neonatal prognosis.

In order to reduce these complications, expectation management can be used, when the situation allows. The conditions that impose emergency therapeutic behavior are divided into maternal and fetal conditions. The maternal conditions include high blood pressure values resistant to antihypertensive treatment (greater than 160/110 $\mathrm{mmHg}$ ); persistent, treatment-resistant headache; epigastralgia or pain in the right shoulder resistant to anti-algic treatment; visual disorders, motor deficits or sensory disorders; stroke; myocardial infarction; HELLP syndrome; newly developed renal failure or worsening of renal function; pulmonary 
edema; eclampsia; and suspected abruptio placentae or vaginal bleeding in the context of the placenta praevia $^{1}$.

Fetal conditions that require emergency therapeutic action include a biophysical score of 4 or less; intrauterine fetal death; minimal chances of fetal survival in the context of fetal malformation incompatible with life or extreme prematurity; changes in Doppler velocimetry with inverted end-diastolic flow of the umbilical artery ${ }^{2}$.

The decision to implement therapeutic intervention is made after the complete clinical and paraclinical evaluation and the determination of the risk/benefit ratio, both maternal and fetal. More specifically, the biological evaluation should be performed prior to obstetrical decision and should include: hemoleucogram; biochemical evaluation of renal function, liver function, and markers of hemolysis; and urinary test for evaluation of proteinuria. The fetal evaluation is based on the complete obstetrical ultrasound examination, with assessment of fetal growth and weight, respectively, as well as assessment of the volume of the amniotic fluid and the fetal biophysical score along with the Doppler velocimetry of the umbilical arteries, the mean cerebral artery and the cerebroplacental ratio ${ }^{3}$. Both prematurity and intrauterine growth restriction are the fetal complications most often associated with preeclampsia. Intrauterine growth restriction is a marker of fetal distress and an important risk factor for fetal intrauterine degradation, and the onset of complications of prematurity further contribute to a less favorable prognosis ${ }^{4}$.

The aim of our study was to evaluate the neonatal prognosis of preterm births with and without growth restriction and term births with growth restriction in order to improve decisional accuracy regarding the termination of pregnancy.

\section{Methods}

The purpose of this study was to comparatively evaluate the neonatal evolution and the rate of short-term neonatal complications of the preterm infants with normal weight for the gestational age and of the preterm infants with low weight for the gestational age, as well as of the term infants small for gestational age (SGA). We carried out a retrospective study using the database of the neonates of the Neonatology Clinic of the Bucharest Emergency University Hospital, hospital with a third degree maternity ward, for a period of 3 years. The cases of preterm birth were selected according to the World Health Organization (WHO) definition of birth before 37 full weeks of gestation. Only live newborn cases were selected and analyzed. The cases of premature newborns were classified in two categories according to the birth weight, namely with appropriate weight for the gestational age, named group 1 and including 78 cases and with low weight according to the gestational age corresponding to the international growth curves, named group 2 and including 1121 case. Therefore, the low weight cases selected were those in which the weight at birth was below the two standard deviations of the growth curves corresponding to the gestational age. There were also cases of babies with low birth weight born at term who were selected for the purpose of comparative analysis of their neonatal prognosis (group 3 including 206 cases). The parameters that were analyzed included gestational weight and age as directional criteria, fetal sex, mode of birth (cesarean or spontaneous birth), and Apgar index at 1 minute as a marker of immediate postnatal fetal status. Meanwhile, the obstetric features analyzed included fetal presentation, the spectrum of the spectrum of hypertensive disorders of pregnancy, fetal malformations, nuchal cord and true umbilical cord knot. The immediate neonatal complications and neonatal markers analyzed included: cardio-vascular arrest, acute respiratory failure, ulcer-necrotic enterocolitis, hypoxia, respiratory distress, cerebral edema, intraventricular hemorrhage, cerebral hemorrhage, pulmonary hemorrhage, patent ductus arteriosus, neonatal hypoglycemia, retinopathy, anemia, hemorrhagic disease, disseminated vascular coagulation, hyaline membrane disease, neonatal sepsis, need for neonatal intensive care, and death. A total of 1405 subjects were analyzed and were divided into 3 study groups: group 1 representing live, preterm infants with low weight for gestational age; group 2 representing living newborns, premature but with weight corresponding to the gestational age; and group representing term newborns with low birth weight. The cumulative and comparative analysis by frequency and sex were analyzed in the first phase, following the cumulative and comparative analysis of all the neonatal and obstetrical parameters of the cases in the 3 groups and the statistical analysis regarding the significant difference, or the frequency of the complications analyzed, in those 3 study groups through the chi-square test. The results were analyzed 
and interpreted according to the obtained $\mathrm{P}$ value; $\mathrm{P}<0.05$ was considered to be statistically significant.

The data collected retrospectively did not contain personal information and only the ethics committee agreement of the University Emergency Hospital of Bucharest was required and obtained without the need of informed consent or the consent of the patient/legal representative in the case of minors.

\section{Results}

Our group of study included a total of 1405 newborns with a minimum gestational age of 23 weeks and a maximum of 41 weeks. Regarding the correspondent birth weight, the minimum value recorded in the total study group was 240 grams and the maximum 4260 grams. In figure 1 is represented the division into groups, according to the working method.

During the duration of the study (3 years), a total of 1199 premature infants were registered, of which the majority (1121 cases, $93.5 \%$ ) had appropriate weight for gestational age, and only $6.5 \%$ of the preterm cases were classified as presenting reduced weight for the gestational age. The frequency of term birth infants with low birth weight for gestational age was approximately $2 \%$ of all births registered during the three years of study.

In the study group, the highest proportion, group $2(79.8 \%)$ had premature infants with normal weight for the gestational age followed by group 3with term infants with low weight for the gestational age (14.7\%), and group 1with preterm infants with low weight for gestational age (5.6\%) (Table 1).

Regarding the frequency per gender, the male gender, noted with 1 in Table 2, predominated only in study group 2, the group of premature infants with normal weight for the gestational age. The other 2 study groups were characterized by low weight for gestational age with predominantly female infants.

\section{Apgar index}

For each study group, the Apgar index was analyzed in terms of median, maximum, and minimum. Thus, for all 3 study groups, the following results were obtained for a $95 \%$ confidence interval. Group 1 had a median Apgar index of 7, with a minimum value of 1 and a maximum value of 9. Group 2 was characterized by a median of the Apgar index of 8, a minimum value of 0 , and a maximum value of 10 . Study group 3 was characterized by a median Apgar index of 9, a minimum value of 1 and a maximum value of 10 (Fig 2).

Regarding the mode of birth, for all the 3 study groups, birth through cesarean section predominated (Table 3). The greatest difference in percentage was in the group of premature infants with low birth weight for the gestational age, with a difference of more than 3 times the percentage $(23 \%)$ for the spontaneous birth and for the cesarean birth $(77 \%)$.

The smallest difference was obtained for the term low birth cases with low weight for the gestational age, with a difference of $16 \%$, favoring birth through caesarean section. For the entire study group, birth by cesarean section predominated, and there was also a statistical significant difference between the 3 study groups, $\mathrm{P}=0.004$.

As we have already mentioned in the subchapter dedicated to the working method, we independently analyzed obstetric and neonatal parameters both from a descriptive point of view and from the statistical differences that exist between the 3 study groups.

The incidence of obstetric aspects, namely fetal presentation, obstetric pathology in the spectrum of hypertensive pregnancy disorders, fetal malformations, multiple nuchal cord, or true umbilical cord knot is summarized in Figure 3.

As can be seen, the incidence of pelvic presentation predominates in pregnancies with low gestational age, that is, in both normal and low-weight premature pregnancies, and hypertensive pregnancy pathology has predominated in study groups characterized by low weight; however, there was no statistical difference for the low birth weight group in our study. 
All the neonatal complications, including the incidence of the complications within each study group, and the existence or not of statistical significance for the study groups are shown in the table below (Table 4).

Analyzing each parameter separately, we obtained a statistically significant difference, predominantly in preterm infants with low weight for the gestational age, for the following complications: cardio-vascular arrest occurred in $6 \%$ of the subjects in group 1, with a significant difference of $1 \%$ in the incidence of complications between groups 2 and 3; for acute respiratory insufficiency, the difference in incidence was even greater, in which the premature infants with low weight for the gestational age (24\% compared to $8 \%$ and $2 \%$ corresponding to the other groups); a difference in the incidence for ulcer-necrotic enterocolitis (group 1, 13\%; group 2, 1\%; and group 3,0\%), having statistical significance was otherwise obtained according to the collected data; hypoxia also predominated among preterm infants: $58 \%$ of infants with low weight for gestational age, $23 \%$ for premature infants with weight corresponding to gestational age, and $9 \%$ for term infants with low weight for gestational age.

Respiratory distress had an incidence of $29 \%$ in group 1, significantly higher than group $2(13 \%)$ and group $3(0 \%)$, and cerebral edema occurred only in premature cases, with an incidence of $6 \%$ for those with low weight for gestational age and $2 \%$ for those with appropriate weight.

Neonatal complications, such as cerebral edema, pulmonary hemorrhage, neonatal seizures and disseminated intravascular coagulation, persistence of the arterial canal, cerebral hemorrhage, hyaline membrane disease, and retinopathy had a $0 \%$ incidence in the term neonatal group with low birth weight. Each of these complications predominated in the group of preterm newborns with intrauterine growth restriction, with the exception of the persistence of the arterial canal, which predominated in study group 2, with an incidence of $1 \%$, and was absent in group 1. For cerebral edema, pulmonary edema and disseminated intravascular coagulation, the difference in incidence was statistically significant. For group 3, complications such as intraventricular hemorrhage and neonatal anemia presented minimum incidences of $1 \%$ and $2 \%$, respectively. For these complications, the predominance was also the highest in group 1 (premature infants with low weight for the gestational age), namely $28 \%$ and $32 \%$, respectively, which are significantly higher percentages than those obtained in the other study groups.

Regarding the incidence of neonatal infections, a significant incidence was noted in all 3 study groups, with a significant predominance among the group of premature infants with low-weight for the gestational age (38\%); meanwhile, for the premature infants with the weight corresponding to the gestational age, the incidence was $19 \%$, similar to that of term newborns with low weight for gestational age (16\%). Regarding neonatal sepsis, a higher incidence was obtained of infants with low birth weight for gestational age (15\%) and significant but smaller incidence obtained for the other 2 study groups ( $6 \%$ and $8 \%$, respectively).

Regarding the need for neonatal intensive care, it was $18 \%$ for group 1, $5 \%$ for group 2 , and $1 \%$ for group 3 , with a statistically significant difference between the 3 groups. Additionally, a statistically significant difference was obtained for the rate of neonatal death, which predominated by $8 \%$ in preterm infants with low weight for gestational age and was absent in term infants with low weight for gestational age.

\section{Discussion}

Prematurity remains the leading cause of neonatal morbidity and mortality. Intrauterine growth restriction is another first-line cause of the adverse neonatal prognosis, both as a single pathology or in association with prematurity. According to studies, there is a concordant relationship between preterm birth and intrauterine growth restriction. ${ }^{5}$ Intrauterine growth restriction shows chronic fetal distress based on placental dysfunction, with placental abnormal vascularization leading to hypoperfusion, ischemia, and release of reactive oxygen species in the context of oxidative stress.

Both prematurity and intrauterine growth restriction have an increased incidence among pregnancies complicated with preeclampsia ${ }^{6}$, results that are consistent with the data in the literature. In this context, we decided to highlight the unfavorable short-term prognosis of prematurity, as well as intrauterine growth restriction, in both preterm and term infants with low birth weight. 
According to the results obtained, the frequency of premature births in the Emergency University Hospital of Bucharest, a multidisciplinary hospital in which multiple complex cases are addressed, was approximately $13 \%$. The cases of complicated preterm infants with intrauterine growth restriction accounted for $6.5 \%$ of all premature births recorded in the clinic during the 3 years of study and about $1 \%$ of all births. The cases of newborns with low birth weight for the gestational age accounted for about $2 \%$ of all births recorded during the study period. Thus, the data obtained are consistent with reports in the literature; the proportion of intrauterine growth restriction among preterm births is higher than that among term births. ${ }^{5}$

Another parameter that was consistent with the published reports is the predominance of female fetal sex in cases of intrauterine growth restriction ${ }^{7,8}$ and the predominance of male sex among premature births. However, the difference was not significant; therefore, according to the results, we can consider the following conclusions of the study by Quinones et al, ${ }^{9}$ focusing mainly on the influence of fetal sex on the perinatal prognosis of cases of intrauterine growth restriction: fetal sex is not associated with unfavorable perinatal prognosis in cases of intrauterine growth restriction.

The purpose of the analysis of the values of the Apgar index as a marker of the immediate neonatal adaptation was to objectively highlight the difference of adaptation, especially in the case of premature infants with low weight for gestational age and those infants with weight corresponding to their gestational age. The values obtained were not different from expectations, more specifically, the adaptation of preterm infants with low weight for the gestational age was the most deficient, with a median value of 7 . Figure 1 shows that there were no values that deviated significantly from the value of the median, with the distribution being proportional. However, the degree of adaptation was higher in the group of premature infants with weight corresponding to the gestational age, as expected. In figure 2, it appears that the values of the Apgar index were higher in this group. However, in the single cases of small Apgar index, the median assigned to group 2 was 8 . The most favorable adaptation was noted in the newborns in group 3. In groups 2 and 3, maximum values of the Apgar score were noted.

Birth by caesarean section predominated in all study groups, with a statistically significant difference noted among the 3 groups. This is justified by the fact that birth by cesarean section of premature infants is associated with lower neonatal mortality ${ }^{10}$, which is explained by the possibility of early and promising neonatal intensive care. ${ }^{11}$ Additionally, in this context, it is worth mentioning that the birth weight is inversely proportional to the rate of neonatal complications, in which the impact of the vaginal birth decreases with increasing fetal weight. ${ }^{12}$ Even though premature birth is not an absolute indication of cesarean delivery, this mode of birth provides a better prognosis for preterm infants by avoiding prolonged labor and allowing for a less traumatic birth. ${ }^{12}$

Regarding obstetric factors, it is not surprising that we obtained a higher incidence of pelvic presentation among preterm infants; however, the incidence of pelvic presentation among low-weight newborns for gestational age was slightly higher than that in the general population. The data at the general population level show an incidence of caesarean section of 4\%-40\% among term births an 25\%-60\% among premature births, which is inversely proportional to the gestational age. As can be seen in Figure 3, the incidence of pregnancy-induced hypertension predominates in cases of growth restriction, both in premature newborns and particularly in newborns with term growth restriction. This situation is also characteristic of preeclampsia, except that it prevails in preterm infants with intrauterine growth restriction and low weight for gestational age. These results confirm the results of other studies, namely that placental functional disorders belong to the group of progressive multifactorial pathologies that present deteriorating signs and symptoms over time.

Fetal malformations predominated in the group of premature newborns with growth restriction, which is consistent with published reports on the association of fetal malformations with intrauterine growth restriction ${ }^{13,14}$ and prematurity. ${ }^{15}$ The umbilical cord pathology, mainly the true cord knot, did not have a significant association with intrauterine growth restriction, which is consistent with the data in the literature; however, this association has not been fully established and is still being studied ${ }^{16,17,18}$. Regarding the multiple nuchal cord, a statistically significant association has not yet been evidenced between these 
condition and adverse neonatal prognosis. ${ }^{19}$ According to the results obtained in our study, the incidence of multiple nuchal cord was higher in the groups with growth restriction.

Essentially, the purpose of our study is to show that intrauterine growth restriction, a condition closely related to placental dysfunction, is a common diagnosis that is associated with an increased risk of perinatal mortality and morbidity. The fetal response consists in circulatory adaptations, respectively brain-sparing reflected by the value of cerebroplacental ratio, which has a better predictability index of adverse outcomes especially in fetuses with intrauterine growth restriction ${ }^{20}$. The meta-analysis published in 2016 , which had the aim to evaluate the perinatal predictability value of cerebroplacental ratio concluded that abnormal cerebroplacental ratio is associated with increased rates of unfavorable perinatal outcome, having a moderatehigh specificity and sensitivity ${ }^{21}$. An abnormal cerebroplacental ratio was associated with higher rates of need of neonatal intensive care and neonatal complications and suggest a poorer perinatal outcome of fetuses with intrauterine growth restriction ${ }^{21}$. Regarding the normalization of the cerebroplacental ratio, the results of a recent sub-analysis which started from the hypothesis that normalization of this ratio associates a poorer perinatal outcome due to the loss of the compensatory mechanism of brain sparing, showed that there is no additional worsening of the perinatal prognosis given by this normalization ${ }^{22}$. Recent studies have analyzed the impact of the abnormal cerebroplacental ratio on neurodevelopmental outcome in fetuses with intrauterine growth restriction. Meher et al in his review suggested that the brain sparing phenomenon has not only a protective benefit but is associated with a poorer psychomotor development at one and two years caused by implied cerebral hypoxia ${ }^{23}$.

In the context of the results obtained, as well as of the discussions regarding the advantages of the expectant management for both short and long-term outcome, the decision regarding choosing the most appropriate time for termination of the pregnancy becomes even more difficult. Thus, each case should be treated individually with a therapeutic behavior guided by the main pathology but also the associated one in order to reduce the rate of iatrogenic prematurity among the fetuses with intrauterine growth restriction but also to offer them the best prognosis.

Further, with reference to Table 4, which contains the frequency and comparative analysis of all the neonatal complications studied for the 3 groups, one can observe the objective impact of intrauterine growth restriction during the immediate neonatal period.

Thus, as discussed in the results chapter, the highest frequency of neonatal complications occurred in study group 1. Statistical significance was obtained for the following complications: cardio-vascular arrest (P $<0.001)$, acute respiratory failure $(\mathrm{P}<0.001)$, ulcer-necrotic enterocolitis $(\mathrm{P}<0.001)$, hypoxia present in $58 \%$ of premature cases with growth restriction and in $23 \%$ of cases of gestational age weight $(\mathrm{P}<0.001)$, respiratory distress $(\mathrm{P}<0.001)$, cerebral edema $(\mathrm{P}=0.004)$, intraventricular hemorrhage $(\mathrm{P}<0.001)$, cerebral hemorrhage $(\mathrm{P}=0.003)$, pulmonary hemorrhage $(\mathrm{P}<0.001)$, neonatal infection $(\mathrm{P}<0.001)$, hypoglycemia $(\mathrm{P}$ $<0.001)$, retinopathy $(\mathrm{P}<0.001)$, anemia $(\mathrm{P}<0.001)$, hemorrhagic disease $(\mathrm{P}=0.002)$, disseminated intravascular coagulation $(\mathrm{P}<0.001)$, disease of hyaline membranes $(\mathrm{P}<0.001)$, neonatal sepsis $(\mathrm{P}=0.002)$, need for intensive neonatal therapy $(\mathrm{P}<0.001)$, and death $(\mathrm{P}<0.001)$.

Intrauterine growth restriction is associated with an increased risk of both antenatal and neonatal complications. There is an increased negative impact on prognosis when fetuses with intrauterine growth restriction are born premature. In our study, newborns with low weight for gestational age had an increased incidence in complications in comparison to newborns with adequate weight, specifically: cardio-vascular arrest, $0.1 \%$ in the general population and $1 \%$ in the present study; acute respiratory failure, $0.45 \%$ in the general population and $2 \%$ in the present study ${ }^{12}$; however, respiratory distress, cerebral edema, ulcer-necrotic enterocolitis, pulmonary hemorrhage, persistence of the arterial canal, cerebral hemorrhage, seizures, retinopathy, hemorrhagic disease, disseminated intravascular disease, and hyaline membrane disease were absent in term infants with low birth weight for gestational age, indicating that these newborns have a good neonatal adaptation by leaving an environment already unfit for their well-being, i.e. intrauterine.

\section{Conclusions}


In conclusion, intrauterine growth restriction can occur both in the context of pre-existing chronic hypertension and in the context of severe preeclampsia. In principle, preeclampsia is so frequently associated with intrauterine growth restriction that the latter has traditionally been included as a feature of preeclampsia, regardless any additional diagnostic criteria. During the study, it was shown that preeclampsia is the main condition that leads to the most severe cases of intrauterine growth restriction. Immediate neonatal adaptation of preterm neonate small for gestational age is more deficient (indicated by lower Apgar index values) than for preterm neonates with appropriate weight for gestational age; the adaptation of preterm neonates, in turn, is more deficient than term newborns with intrauterine growth restriction. The term newborns with intrauterine growth restriction have a neonatal adaptation comparable to that of the term newborns with weight corresponding to the gestational age. Birth by caesarean section had an increased incidence both in the cases of premature newborns with weight corresponding to the gestational age and in the cases with premature or term growth restriction. Gestational hypertension is a major risk factor for intrauterine growth restriction without a statistically significant difference between premature and term births. Preeclampsia, on the other hand, is significantly associated with prematurity and intrauterine growth restriction and especially in cases presenting both conditions simultaneously. Fetal malformations are a determinant factor of growth restriction, but also of prematurity.

After analyzing the neonatal parameters of the3 study groups, which included premature infants with low weight for gestational age, preterm infants with weight corresponding to the gestational age and term newborns with low weight for gestational age, we can conclude that the growth restriction superimposed on prematurity is associated the most unfavorable prognosis among all the parameters.

\section{References}

[1] Turcan N, Bohîlțea R, Neacsu A, Baros Al, Cîrstoiu MM. The role of anticoagulant therapy in the prevention of preeclampsia. Pharmacokinetic and pharmacodinamic mechanisms. REV CHIM 2019, 70(4): 1424-1428.

[2] Trăistaru VA, Zamfirescu V, BurneiRusu A, Bohîlțea R, Nastasia Ș, Vlădăreanu R. Ultrasound Markers in Early Pregnancy Predictive for Preeclampsia. 5TH ROMANIAN CONGRESS OF THE ROMANIAN SOCIETY OF ULTRASOUND IN OBSTETRICS AND GYNECOLOGY 2017 : 593-597.

[3] Trăistaru VA, BurneiRusu A, Zamfirescu V, Nastasia Ș, Bohîlțea R, Vlădăreanu R. Ultrasound Markers in Early Pregnancy Predictive for Preterm Birth. 5TH ROMANIAN CONGRESS OF THE ROMANIAN SOCIETY OF ULTRASOUND IN OBSTETRICS AND GYNECOLOGY 2017 : 598-602.

[4] Ionescu AC, Popescu I, Banacu M, Matei A, Bohîlțea R, Dimitriu M. Is it Possible to Predict Stillbirth in the Third Trimester?

5TH ROMANIAN CONGRESS OF THE ROMANIAN SOCIETY OF ULTRASOUND IN OBSTETRICS AND GYNECOLOGY 2017

[5] Bukowski R, Gahn D, Denning J, et al. Impairment of growth in fetuses destined to deliver preterm. Am J Obstet Gynecol. 2001;185:463-467.

[6] Bohîltea R, Turcan N, Ionescu C, Toader O, Nastasia S, Neculcea D, Movileanu I, Munteanu O, Cîrstoiu M. The Incidence of Prematurity and Associated Short-Term Complications in a Multidisciplinary Emergency Hospital from Romania. 5TH ROMANIAN CONGRESS OF THE ROMANIAN SOCIETY OF ULTRASOUND IN OBSTETRICS AND GYNECOLOGY 2017: 105-112.

[7] Spinillo A, Capuzzo E, Nicola S, et al. Interaction between fetal gender and risk factors for fetal growth retardation. Am J Obstet Gynecol. 1994;171:1273-1277.

[8] Yunis KA, Beydoun H, Tamin H, et al. Risk factors for term or near-term fetal growth restriction in the absence of maternal complications. Am J Perinatol. 2004;21:227-234. 
[9] Quinones JN, Stamilio DM, Coassolo KM, et al. Is fetal gender associated with adverse perinatal outcome in intrauterine growth restriction (IUGR)? Am J Obstet Gynecol. 2005;193:1233-1237.

[10] Bohîlțea RE, Zugravu CA, Neacsu A, Navolan D, Berceanu C, Nemescu D, Bodean O, Turcan N, Baros Al, Cîrstoiu MM. The prevalence of Vitamin D deficiency and its obstetrical effects. A prospective study on Romanian patients. REV CHIM 2019, 70 (4): 1228-1233.

[11] Dobson PC, Abell DA, Beischer NA. Mortality and morbidity of fetal growth retardation. Aust $N Z J$ ObstetGynaecol. 1981;21:69-72.

[12] Ment LR, Oh W, Phillip AG, et al. Risk factors for early intraventricular hemorrhage for low birth weight infants. J Pediatr. 1992;121:776-783.

[13] Resnik R. Intrauterine growth restriction. Obstet Gynecol. 2002;99:490-496.

[14] Herghelegiu D, Ionescu CA, Pacu I, Bohîlțea R, Herghelegiu C, Vladareanu S. Antenatal diagnosis and prognostic factors of aneurysmal malformation of the vein of Galen. A case report and literature review.Medicine (2017) 96:30(e7483)

[15] Purisch SE, DeFranco EA, Muglia LJ, et al. Preterm birth in pregnancies complicated by major congenital malformations: a population-based study. Am J Obstet Gynecol. 2008;199:287.e1-287.e8.

[16] Marin JA, Calomfirescu M, Bohilțea R, Ionescu Târgoviște C. Impact of Maternal and Placental Pathology on Successful Umbilical Cord Blood Sampling and Cryopreservation. Gineco.ro 2011; 7(23/1):10-14.

[17] Szczepanik ME, Wittich AC. True knot of the umbilical cord: a report of 13 cases. Mil Med. 2007;172:892-894.

[18] Schreiber H, Daikan Y, Arbib N, et al. Multiple nuchal cord loops and neonatal outcomes. AJOG. 2018;218:S91

[19] Rubaltelli FF, Dani C, Reali MF, et al. Acute neonatal respiratory distress in Italy: a one-year prospective study. Italian Group of Neonatal Pneumology. ActaPaediatr. 1998;87:1261-1268.

[20] Bahado-Singh RO, Kovanci E, Jeffres A, et al. The Doppler cerebroplacental ratio and perinatal outcome in intrauterine growth restriction. Am J ObstetGynecol 1999; 180: 750-756.

[21] Nassr, A. A., Abdelmagied, A. M., \&Shazly, S. A. M.. Fetal cerebro-placental ratio and adverse perinatal outcome: systematic review and meta-analysis of the association and diagnostic performance. Journal of Perinatal Medicine 2016; 44(2): 249-256.

[22] Monteith, C., Flood, K., Mullers, S., Unterscheider, J., Breathnach, F., et al. Evaluation of normalization of cerebro-placental ratio as a potential predictor for adverse outcome in SGA fetuses. American Journal of Obstetrics and Gynecology 2017, 216(3), 285.e1-285.e6

[23] Meher, S., Hernandez-Andrade, E., Basheer, S. N., \& Lees, C. Impact of cerebral redistribution on neurodevelopmental outcome in small-for-gestational-age or growth-restricted babies: a systematic review. Ultrasound in Obstetrics \& Gynecology 2015, 46(4), 398-404.

\section{Hosted file}

Figures.docx available at https://authorea.com/users/293135/articles/421037-the-unfavorableinfluence-of-the-prematurity-on-the-neonatal-prognostic-of-small-for-gestational-agefetuses

\section{Hosted file}

Tables .docx available at https://authorea.com/users/293135/articles/421037-the-unfavorableinfluence-of-the-prematurity-on-the-neonatal-prognostic-of-small-for-gestational-age-

fetuses 